\title{
Alternativas para seleção de touros da raça Nelore considerando características múltiplas de importância econômica
}

[Nellore bull selection alternatives using multiple traits of economic importance]

\author{
J.E. Val $^{1}$, A.S. Ferraudo ${ }^{2}$, L.A. F. Bezerra ${ }^{1}$, M.P. Corrado ${ }^{1}$, R.B. Lôbo $^{1}$, \\ M.A.R. Freitas ${ }^{1}$, J.C.C. Paneto ${ }^{1}$ \\ ${ }^{1}$ Faculdade de Medicina de Ribeirão Preto - USP - Ribeirão Preto, SP \\ ${ }^{2}$ Faculdade de Ciências Agrárias e Veterinárias - UNESP - Jaboticabal, SP
}

\begin{abstract}
RESUMO
Os dados são provenientes de 234 touros da raça Nelore participantes de um teste de progênie, no período de 1996 a 2003. A diferença esperada na progênie (DEP) de sete características: peso aos 120 e 210 dias, efeito materno (DMPP120 e DMPP210), peso e perímetro escrotal aos 365 e 450 dias, efeito direto (DDP365, DDP450, DDPE365 e DDPE450) e idade ao primeiro parto (DDIPP) foi utilizada para classificar os animais em três grupos, assim como identificar quais as características possuíram maior poder discriminatório na formação de cada grupo. Para tanto, foram utilizados procedimentos estatísticos multivariados de análise de agrupamentos k-médias e componentes principais. Os resultados evidenciaram que, dos três grupos formados, dois se destacaram quanto aos valores médios das DEPs. A importância desses dois grupos de touros foi confirmada pela análise de componentes principais, que associou a eles valores superiores de DEPs diretas de peso e perímetro escrotal. A quantidade da variabilidade original retida pelos dois primeiros componentes principais foi de 70,22\%. Estes procedimentos mostraram-se eficientes e constituíram importantes ferramentas para classificar touros, discriminar variáveis, bem como resumir informações multivariadas, podendo ser usados como auxílio valioso na seleção de reprodutores para uso nos programas de melhoramento genético.
\end{abstract}

Palavras-chave: melhoramento genético, teste de progênie, classificação de touro, componente principal

\begin{abstract}
The data set is from 234 Nelore bulls which participate of a progeny test in the period from 1996 to 2003. The expected progeny diference (EPDs) of seven economic traits, weights at 120 e 210 days of age, maternal effect (DMPP120 and DMPP210), weights and scrotal circumferences at 365 e 450 days of age, direct effects (DDP365, DDP450, DDPE365 and DDPE450), and age at first calving (DDIPP), were used in order to classify these animals in three groups and verify which EPDs showed greatest discriminating power in forming the groups. The statistical applied techniques were: $k$-means clusters analysis and principal components analysis. From the three groups formed, two of them stood out in relation to values of the EPDs means. Evidence of the importance of these two groups was observed in the principal component analysis that associate to them higher values of direct EPDs of weight and scrotal circumference. The two first principal components accounted for $70.22 \%$ of the total original variability. Both techniques could be an important tools to sire classification, variable discrimination, as well as to resume multivariate information, and they could be applied to help selection in animal breeding program.
\end{abstract}

Keywords: breeding program, progeny test, sire classification, principal component

Recebido em 17 de outubro de 2006

Aceito em 12 de março de 2008

E-mail: jedval2@hotmail.com

Apoio: CAPES, ANCP, PRONEX, FAPESP 


\section{INTRODUÇÃO}

Os programas de melhoramento genético de bovinos das diversas raças, juntamente com as ações de outras áreas envolvidas nos sistemas de produção, assumem papel relevante no incremento da produtividade da bovinocultura de corte brasileira. A limitação na identificação dos melhores animais para reprodução reside no fato de que o mérito genético não é mensurável diretamente, portanto deve ser predito. A diferença esperada na progênie (DEP) representa a metade do valor genético obtido nas avaliações genéticas. Ela é usada para comparar os méritos genéticos de animais em várias características e predizer a habilidade de transmissão genética do animal avaliado como progenitor (Lôbo et al., 2003).

Segundo Tonhati et al. (2003), o resultado final da exploração dos bovinos de corte depende de várias características; assim, normalmente é necessário que se trabalhe a seleção em várias direções, a fim de se melhorar o desempenho geral dos rebanhos. $\mathrm{O}$ aprimoramento das técnicas de avaliação genética tem gerado número cada vez maior de informações, disponibilizando DEPs para diferentes características, o que tem dificultado os processos de tomada de decisão na seleção.

A tecnologia computacional hoje disponível permite avanços extraordinários na análise de dados e o uso das técnicas analíticas multivariadas por pesquisadores, nas diferentes áreas da indústria e em centros de pesquisa acadêmica é crescente. A análise multivariada pode ser definida como método estatístico que analisa simultaneamente múltiplas medidas sobre a unidade experimental. As variáveis devem ser aleatórias e inter-relacionadas, de maneira que seus efeitos não possam ser significativamente interpretados de forma separada (Hair et al., 2005). Para Cardoso et al. (2003), as análises de agrupamento e os componentes principais são procedimentos multivariados que podem auxiliar a utilização das diferentes DEPs e maximizar os resultados obtidos com seleção. Esses autores utilizaram o método k-médias para separar grupos homogêneos de reprodutores múltiplos de bovinos da raça Nelore.

A análise de agrupamento utiliza métodos hierárquicos e não-hierárquicos, ambos são não- supervisionados e extraem propriedades estatísticas de um conjunto de dados, agrupando os vetores similares em classes. Um método não-hierárquico bastante utilizado é o k-médias, que classifica objetos em número predefinido de grupos. A medida de similaridade usada entre os vetores de médias dos grupos pode levar a diferentes formações quanto à composição e ao número de objetos dentro de cada grupo. Assim, a escolha dessa medida deve observar critérios, sendo a distância euclidiana um dos mais utilizados, por ser uma métrica completa (Hair et al., 2005).

A análise de componentes principais é outra técnica multivariada, que reduz a quantidade de variáveis originais num conjunto menor, preservando o máximo da variabilidade original. Esta técnica cria eixos ortogonais, que são combinações lineares das variáveis originais, partindo dos autovalores da matriz de covariância das variáveis consideradas. Os dois maiores autovalores geram os dois primeiros componentes principais, que agregam maior quantidade de variabilidade que qualquer um dos outros componentes (Johnson e Wichern, 1992).

A genética quantitativa tem mostrado três direcionamentos para uso dos componentes principais (CPs) como ferramenta para visualizar padrões de variação genética, para definir parâmetros genéticos a serem estimados e para separar o número original de variáveis até um conjunto menor de componentes principais fenotípicos e, então, estimar os parâmetros genéticos destes CPs (Kirkpatric e Meyer, 2004).

Mascioli et al. (2000) aplicaram a técnica dos componentes principais (CPs) em características de crescimento, pesos e ganhos de peso em animais da raça Canchim e concluíram que 75\% da variabilidade nas variáveis originais foram retidas nos três primeiros componentes principais, sendo que os pesos à desmama e aos 24 meses e o ganho de peso entre essas duas pesagens obtiveram maior destaque nas análises.

Cardoso et al. (2003) utilizaram componentes principais para analisar dois conjuntos de dados contento DEPs de nove características pré e pósdesmama de um mesmo rebanho da raça Nelore: um conjunto contendo 4740 vacas e outro contendo 
158 touros. Segundo os autores, os três primeiros componentes principais explicaram $76 \%$ e $71 \%$ da variabilidade genética contida nos conjuntos de dados originais, respectivamente para vacas e touros. Quanto ao conjunto de vacas, foi possível detectar alta variabilidade em precocidade e discriminar dois grupos de animais, um contendo alta qualidade genética para características até a desmama e outro contendo alta qualidade genética para características no pós-desmama; também foram encontradas diferenças em precocidade sexual. Em relação ao conjunto de touros, Cardoso et al. (2003) discutem que a interpretação dos resultados foi mais complexa por conseqüência da alta pressão de seleção, mesmo assim foi possível discriminar características à desmama com características ao sobreano, bem como precocidade de terminação, musculosidade e perímetro escrotal.

Visando facilitar a escolha de reprodutores quando características múltiplas são consideradas, este trabalho teve como objetivo identificar, por meio de abordagens multivariadas, grupos de animais cujas DEPs apresentem padrões de semelhança, assim como discriminar as variáveis que mais influenciaram na divisão dos grupos, para auxiliar as decisões na seleção de bovinos de corte em programas de melhoramento genético e maximizar a eficiência da produção.

\section{MATERIAL E MÉTODOS}

Neste estudo foram utilizadas informações de touros da raça Nelore provenientes de rebanhos participantes do Programa de Melhoramento Genético da Raça Nelore (PMGRN-Nelore Brasil). Entre as tecnologias geradas, o programa desenvolve, desde 1995, um teste de progênie de touros jovens denominado Reprodução Programada (RP), que tem como finalidade identificar e testar precocemente reprodutores jovens, bem como multiplicar este material genético e gerar, posteriormente, avaliações genéticas mais confiáveis.

Este estudo envolveu informações de 234 touros participantes da RP realizadas no período de 1996 a 2003. As variáveis utilizadas foram as DEPs das características de peso aos 120 e 210 dias de idade, efeito materno (DMPP120 e DMPP210), peso e perímetro escrotal aos 365 e 450 dias de idade, efeito direto (DDPP365, DDPP450, DDPE365 e DDPE450) e idade ao primeiro parto, efeito direto (DDIPP). Os valores das DEPs foram obtidos na avaliação genética de 2003, utilizando a metodologia dos modelos mistos (BLUP), sob modelo animal completo, pela aplicação dos softwares MTDFREML e TKBLUP (Boldman et al., 1995; Golden et al., 1995).

A partir dos resultados da avaliação genética, foram estabelecidos critérios para pré-seleção dos touros candidatos a participar do teste de progênie. Assim, foram incluídos machos com idades entre 12 e 36 meses, que não apresentavam valores negativos para DMPP120, DDPP365 e DDPE365, pertenciam a grupos de contemporâneos com número de indivíduos maior ou igual a cinco e estavam classificados entre os 3\% superiores para mérito genético total (MGT), índice de seleção utilizado pelo PMGRN.

Seqüencialmente a essa pré-seleção, foram feitas as avaliações fenotípicas dos animais nas fazendas, realizadas por equipe técnica previamente estabelecida e treinada. Finalmente, os animais selecionados foram encaminhados às centrais de inseminação artificial (IA) para colheita do sêmen distribuído para as fazendas participantes.

Os procedimentos multivariados, k-médias e componentes principais, foram aplicados às referidas DEPs com valores padronizados, resultando em média nula e variância unitária (Hartingan, 1975). A não-padronização poderia levar a inconsistências nas soluções das duas técnicas, já que a maioria das medidas de distância é bastante sensível a diferentes escalas ou magnitudes das variáveis. Essas análises estatísticas foram classificadas no contexto das análises multivariadas como técnicas de interdependência, nas quais nenhuma variável é definida como independente ou dependente, pois o processo envolve a análise simultânea de todas as variáveis em conjunto, e foram realizadas com o auxilio do software Statsoft (Data..., 2004).

$\mathrm{O}$ método k-médias busca a melhor solução na divisão de grupos, de tal modo que a semelhança dentro de grupos e as diferenças entre grupos sejam máximas (Reis, 2001; Pérez, 2001; Hair et al., 2005).

O algoritmo geral do método pode ser assim descrito:

a) escolher $k$ distintos valores para centros dos grupos. Podem ser utilizadas $k$ sementes 
aleatórias para assumir cada centro inicial dos grupos, ou pode-se usar uma pequena parte dos dados para calcular centros iniciais dos grupos; b) associar cada ponto ao centro mais próximo; c) recalcular o centro de cada grupo;

d) repetir os passos b) e c) até não haver alterações.

Das tentativas realizadas para a formação de grupos de animais com DEPs similares, $\mathrm{k}=3$ foi o valor que melhor representou uma estrutura classificatória dos touros em grupos. Como medida de similaridade entre os animais, foi utilizada a distância euclidiana num espaço de sete dimensões, que corresponde às DEPs das sete características estudadas.

A distância euclidiana num espaço multidimensional pode ser assim descrita:

$\mathrm{d}(\mathrm{x}, \mathrm{y})=\left\{\mathrm{S}_{\mathrm{i}}\left(\mathrm{x}_{\mathrm{i}}-\mathrm{y}_{\mathrm{i}}\right)^{2}\right\}^{1 / 2}$; em que:

$\mathrm{d}(\mathrm{x}, \mathrm{y})=$ distância entre objetos $\mathrm{x}$ e $\mathrm{y}$

$\mathrm{S}_{\mathrm{i}}=$ somatório de $\mathrm{i}$ dimensões

A partir dos grupos gerados pelo método de agrupamento k-médias, os touros foram codificados conforme o grupo pertencente $(1,2$ ou 3) e, então, foi aplicada a técnica de componentes principais (Hair et al., 2005), utilizando as mesmas DEPs citadas inicialmente, com o intuito de visualizar a distribuição dos grupos de touros no plano bidimensional formado por componentes principais bem como interpretar o poder discriminatório das DEPs dessas características em cada componente principal, conforme:

$r_{x j}\left(C P_{h}\right)=\frac{a_{j h} \sqrt{\lambda_{h}}}{S_{j}}$; em que:

$S_{j}=$ desvio-padrão da variável $j$;

$a_{j h}=$ coeficiente da variável j no h-ésimo componente principal;

$\lambda_{h}=$ autovalor $\mathrm{h}$;

$r_{x j}\left(C P_{h}\right)=$ correlação da variável $\mathrm{x}_{\mathrm{j}}$ com o $\mathrm{h}$ ésimo componente principal.

Os autovetores $\left(\mathrm{CP} 1, \mathrm{CP} 2, \ldots ., \mathrm{CP}_{\mathrm{h}}\right)$ foram construídos a partir dos autovalores da matriz de covariância das DEPs em ordem decrescente; sendo assim, o CP1 é o componente que retém mais variabilidade do conjunto original dos dados, enquanto o último componente retém menos.

A variância retida em cada componente principal pode ser calculada da seguinte forma:

$\mathrm{CP}_{\mathrm{h}}=\frac{\lambda_{\mathrm{h}}}{\overrightarrow{\mathrm{t}} \operatorname{raço}(\mathrm{C})} \times 100$; em que:

$C P_{h}=$ componente principal $h$;

$\lambda_{h}=$ autovalor $h$;

$C=$ matriz de covariância e

$\operatorname{traço}(C)=\lambda_{1}+\lambda_{2}+\ldots \ldots . .+\lambda_{h}$.

Ao conjunto de dados utilizado, foram incluídos seis touros, GM, LD, GO, RG, ZF e controle, com o objetivo de auxiliar na interpretação dos grupos formados. Os touros GM, LD e GO são conhecidos como genearcas nacionais da raça Nelore e RG, ZF e controle foram incluídos por representarem os extremos da amostra.

\section{RESULTADOS E DISCUSSÃO}

Na Fig. 1, observa-se o perfil das médias das DMPP120, DMPP210, DDPP365, DDPP450, DDPE365, DDPE450 e DDIPP em cada um dos três grupos definidos. As médias das DEPs do grupo 1 foram inferiores em relação aos grupos 2 e 3 , sendo que estes dois últimos grupos apresentaram comportamentos distintos entre as DEPs das características maternas de peso aos 120 e 210 dias de idade e das características diretas de peso e perímetro escrotal aos 365 e 450 dias de idade. Entre esses, o grupo 2 foi o que apresentou menores valores de médias para DEPs maternais e valores mais altos para as DEPs diretas de peso, fato que pode fornecer condições para melhor escolha quanto à habilidade materna ou de crescimento dos animais, de acordo com os interesses e objetivos de seleção.

$\mathrm{Na}$ Tab. 1, encontram-se os resultados da análise de variância em que se testou a significância das DEPs em cada grupo separado pelo método kmédias. Observou-se que todas as DEPs apresentaram diferenças significativas entre grupos $(\mathrm{P}<0,0005)$.

A distribuição dos touros, de acordo com os dois primeiros componentes principais (CP1 e CP2), seguiu o mesmo padrão de classificação dos três 
grupos obtidos pela análise de agrupamento. Constatou-se que a soma da variabilidade retida nestes componentes explicou 70,22\% da variabilidade original, sendo que CP1 e CP2 retêm, cada um, $44,91 \%$ e $25,42 \%$, respectivamente. Esses resultados foram semelhantes aos citados por Cardoso et al. (2003).

Os valores das correlações entre cada DEP e os dois primeiros componentes principais são apresentados na Tab. 2. Verifica-se no CP1 alto poder discriminatório das DEPs diretas para as características de peso e perímetro escrotal, que obtiveram correlações de $-0,85,-0,85,-0,77$ e 0,76, para DDPP365, DDPP450, DDPE365 e DDPE450, respectivamente. No CP2 o poder discriminatório das DEPs das características maternas de peso apresentou correlações de 0,86 para DMPP120 e -0,84 para DMPP210.
Na Fig. 2, encontra-se a distribuição dos touros no plano formado pelos dois primeiros componentes principais (CP1 e $\mathrm{CP} 2)$ e codificados segundo os grupos obtidos pelo método k-médias. Na horizontal (CP1), é contrastante o posicionamento entre os grupos 1 (à direita) e 2 (à esquerda). As DEPs com maior poder discriminatório no primeiro componente principal foram: DDPP365 e DDPP450 (correlações próximas de -0,85) e DDPE365 e DDPE450 (correlações próximas de -0,77). Como essas correlações são negativas, indicam que quanto mais um touro do grupo 2 se posicionar à esquerda no eixo horizontal (CP1), maior será o valor dessas DEPs. Embora com pouco poder discriminante, a única correlação positiva $(0,36)$ foi com a DDIPP, indicando que quanto mais um touro se localizar à direita, no eixo horizontal, maiores serão os valores dessa DEP. Então, a interpretação seria de que touros localizados à esquerda apresentam bons valores genéticos para essas características.

Tabela 1. Análise de variância entre os três grupos criados pelo método k-médias

\begin{tabular}{lcccccc}
\hline Característica & SQ-entre grupos & gl & $\begin{array}{c}\text { SQ-dentro de } \\
\text { grupos }\end{array}$ & gl & F & P \\
\hline DMPP120 & 63,3717 & 2 & 174,6282 & 236 & 42,821 & $<0,0005$ \\
DMPP210 & 72,5104 & 2 & 165,4896 & 236 & 51,702 & $<0,0005$ \\
DDPP365 & 112,5826 & 2 & 125,4174 & 236 & 105,924 & $<0,0005$ \\
DDPP450 & 112,3858 & 2 & 125,6142 & 236 & 105,573 & $<0,0005$ \\
DDPE365 & 143,1040 & 2 & 94,8960 & 236 & 177,944 & $<0,0005$ \\
DDPE450 & 150,1423 & 2 & 87,8577 & 236 & 201,653 & $<0,0005$ \\
DDIPP & 13,8869 & 2 & 244,1131 & 236 & 7,311 & 0,0008 \\
\hline
\end{tabular}

DMPP120 e DMPP210: DEP materna de peso aos 120 e 210 dias de idade (kg), respectivamente; DDPP365, DDPP450, DDPE365 e DDPE450: DEP direta de peso e perímetro escrotal aos 365 e 450 dias de idade $(\mathrm{kg} \mathrm{e} \mathrm{cm})$, respectivamente; DDIPP: DEP direta de idade ao primeiro parto (meses); gl: graus de liberdade; F: valor de F; P: probabilidade; SQ: soma de quadrados.

Tabela 2. Correlações entre as DEPs das características e os componentes principais 1 e 2

\begin{tabular}{lcc}
\hline Características & Componente principal 1 & Componente principal 2 \\
\hline DMPP120 & $-0,42$ & $-0,86$ \\
DMPP210 & $-0,47$ & $-0,84$ \\
DDPP365 & $-0,85$ & 0,09 \\
DDPP455 & $-0,85$ & 0,07 \\
DDPE365 & $-0,77$ & 0,39 \\
DDPE455 & $-0,76$ & 0,40 \\
DDIPP & 0,36 & 0,04 \\
\hline
\end{tabular}

DMPP120 e DMPP210: DEP materna de peso aos 120 e 210 dias de idade (kg), respectivamente; DDPP365, DDPP450, DDPE365 e DDPE450: DEP direta de peso e perímetro escrotal aos 365 e 450 dias de idade $(\mathrm{kg} \mathrm{e} \mathrm{cm})$, respectivamente; DDIPP: DEP direta de idade ao primeiro parto (meses). 


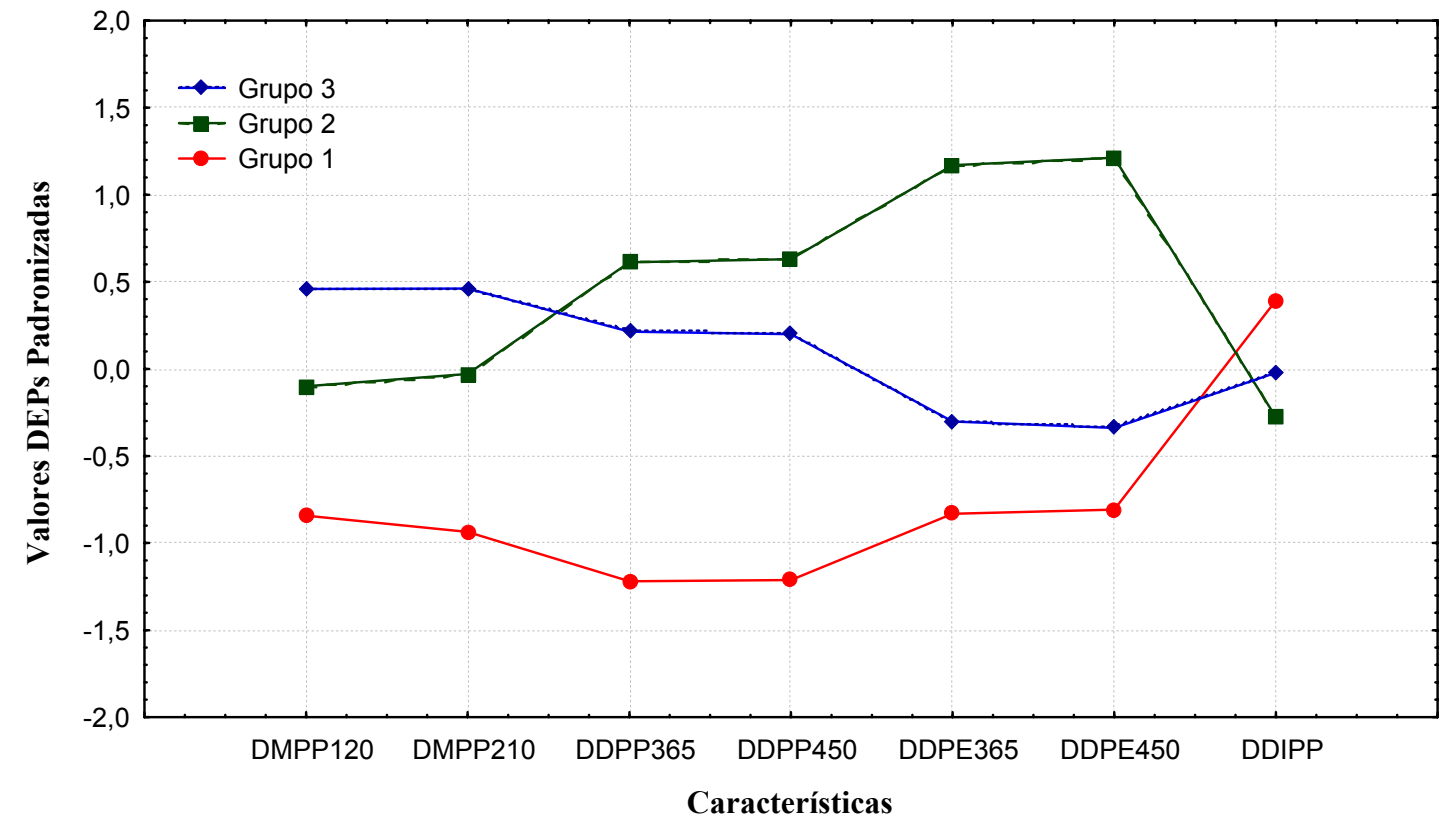

Figura 1. Perfil de médias das DEPs de cada característica e para os três grupos formados pelo método kmédias.

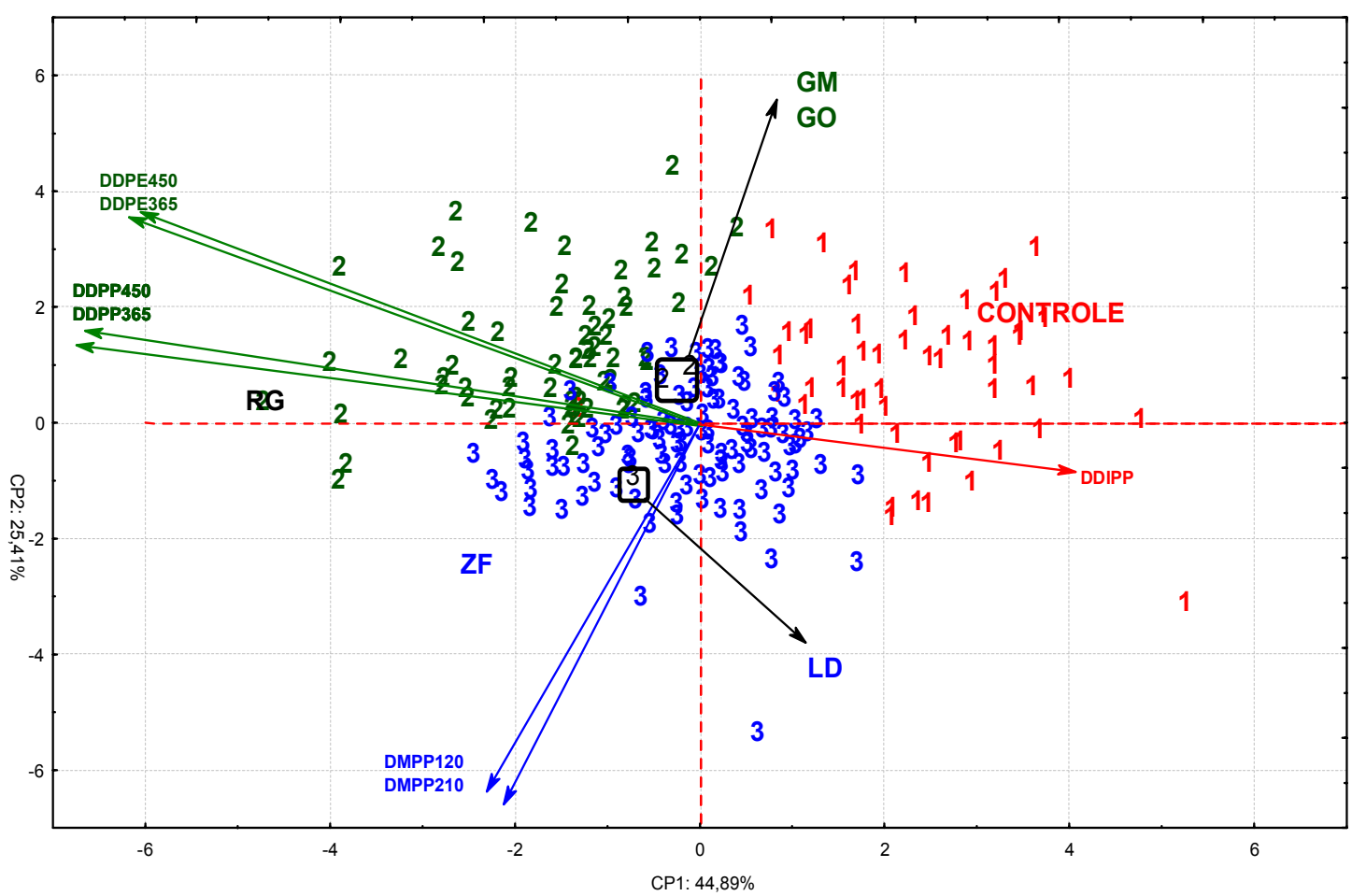

Figura 2. Distribuição dos touros de acordo com os componentes principais 1 e 2 e os vetores das variáveis. 
Quanto ao segundo componente principal, as DEPs discriminatórias estão relacionadas ao efeito materno DMPP120 e DMPP210, tendo correlações negativas $(-0,86$ e $-0,84$, respectivamente). Assim, quanto mais abaixo for a posição de um touro, maiores serão os valores dessas DEPs. Ainda na Fig. 2, observam-se os vetores obtidos na análise de componentes principais, em que pode ser notada uma associação íntima do grupo 2 com as DEPs diretas de peso e perímetro escrotal, enquanto o grupo 3 está associado às DEPs maternas de peso, e o grupo 1 encontra-se associado à DEP da característica idade ao primeiro parto, embora essa associação seja fraca. Isso confirma as correlações obtidas entre as DEPs das características e os referidos componentes.

Na Fig. 2, observa-se também a localização dos animais tomados como referência, codificados como RG, GM, LD, GO, ZF e controle (Lôbo et al., 2003), que foram analisados em conjunto com os 234 touros da pesquisa. Os animais RG, $\mathrm{ZF}$ e controle posicionaram-se em grupos específicos: o RG na horizontal e na esquerda, tendo valores de DEPs diretas de peso e perímetro escrotal semelhantes aos touros dos grupos 2; o controle na horizontal e à direita, possuindo valores de DEPs semelhantes aos touros do grupo 1; e o touro ZF na horizontal à esquerda e na vertical abaixo, possuindo valores de DEPs semelhantes aos touros do grupo 3.

Os touros GM, GO e LD localizaram-se próximos à origem $(0.0)$, não possuem características genéticas discriminatórias considerando as variáveis analisadas, sugerindo que estão envolvidos geneticamente com todos os touros. Isto foi observado também por Vozzi (2004) que, estudando o parentesco entre esses mesmos touros com os principais genearcas da raça Nelore, observaram que o touro LD apresentou parentesco de $95 \%$ com amostra analisada.

Assim, a análise de componentes principais confirma e complementa a divisão realizada pela análise de agrupamento (Fig. 1), em que os touros dos grupos 2 e 3 tendem a possuir qualidades genéticas superiores em relação aos touros que constituem o grupo1.

Na Fig. 2, observa-se, ainda, que o touro RG, conhecido por suas qualidades genéticas superiores no Programa-Nelore Brasil e por se posicionar no extremo de um eixo, possui características discriminatórias relevantes. Esse animal está localizado dentro do grupo 2, conseqüentemente, pode-se concluir que, nesse grupo, cada touro próximo a ele apresente características genéticas semelhantes, o que contribuiria na classificação de reprodutores e auxiliaria nos programas de seleção em bovinos de corte. A mesma discussão é válida para o touro $\mathrm{ZF}$ e grupo 3, assim como para touro controle e grupo 1 .

Convém salientar que quanto mais à esquerda ou à direita, na horizontal, e mais acima ou mais abaixo, na vertical, mais discrepante é o animal quanto às DEPs analisadas. Assim, os animais localizados nos extremos dos eixos na Fig. 2 devem ser preferidos ou excluídos em processos de seleção de touros. Essa análise ainda fornece suporte teórico para se optar por animais de menor custo, mas com conjunto de valores de DEPs similares.

Outra consideração a ser feita é que os touros utilizados foram pré-selecionados de acordo com critérios de seleção compostos em um índice (Lôbo et al., 2003), sendo animais de potencial genético superior, ideais para estabelecimento de padrões de semelhança, o que contribuiu, provavelmente, para a qualidade dos resultados das análises estatísticas efetuadas nesse estudo.

Para os profissionais envolvidos com os programas de melhoramento genético de bovinos, a escolha dos melhores reprodutores não é fácil devido ao acúmulo de variáveis disponíveis. Assim, os procedimentos multivariados, pelas suas qualidades em resumir as informações pósavaliação genética, podem contribuir substancialmente para a seleção dos animais.

É importante mencionar que os resultados obtidos pelos métodos k-médias e componentes principais encontrados neste estudo dependem das suposições de qualidade das DEPs obtidas nas avaliações genéticas. Apesar disso, estes procedimentos podem constituir uma boa ferramenta de auxílio na seleção de reprodutores. Outro fator importante é a acurácia não considerada nessas análises. Ao comparar touros dentro de um mesmo grupo 1, 2 ou 3, deve-se ponderar também os questionamentos habituais quanto à confiabilidade das DEPs. 
Essas técnicas classificatórias poderiam ser aplicadas também para quaisquer grupos de touros ou outros animais, cujos objetivos sejam a separação em lotes homogêneos, como no caso de leilões, venda de animais, separação de linhagens, usos específicos em determinados rebanhos ou para separar grupos ou lotes de manejo. Assim, as técnicas classificatórias podem ser aplicadas, utilizando-se inúmeras variáveis, como peso, sexo, idade, níveis de energia na nutrição, entre outras, além das DEPs como as usadas neste estudo.

Ainda sobre o potencial de uso dessas técnicas para otimização dos trabalhos na área do melhoramento genético nos bovinos de corte, elas podem auxiliar na definição de acasalamentos, em que poderiam ser formados grupos balanceados de fêmeas e machos, de forma a corrigir ocasionais deficiências de certa característica dentro de um grupo. Por exemplo, para um determinado grupo de fêmeas que possuam DEPs maternas baixas, poder-se-iam indicar acasalamentos com grupos de touros que compõem DEPs maternas altas, a fim de equilibrar tal deficiência.

\section{CONCLUSÕES}

Os resultados obtidos nesta pesquisa permitem concluir que as técnicas multivariadas de agrupamento k-médias e os componentes principais mostraram-se eficientes para a classificação dos animais em grupos, identificando padrões de semelhança, sendo que as DEPs de maior destaque na separação dos grupos foram as de peso e perímetro escrotal aos 365 e 450 dias de idade. Os procedimentos multivariados também foram eficientes para resumir as informações após a avaliação genética, promovendo maior facilidade na identificação dos animais mais adequados para determinados rebanhos ou sistemas de produção. Essas técnicas multivariadas podem ser incorporadas às demais ferramentas utilizadas na seleção de reprodutores e no direcionamento dos acasalamentos, entretanto necessitam ser mais testadas e confirmadas em outras pesquisas.

\section{REFERÊNCIAS BIBLIOGRÁFICAS}

BOLDMAN, K.G.A.; KRIESE, L.D.; VAN VLECK, C.P. et al. A manual for use of MTDFREML. A set of programs to obtain estimates of variance and covariances. Washington: USDA ARS, 1995. 120p.
CARDOSO V.; ROSO V.M.; SEVERO J.L.P. et al. Formando lotes uniformes de reprodutores múltiplos e usando-os em acasalamentos dirigidos, em populações Nelore. Rev. Bras. Zootec., v.32, p.834-842, 2003.

DATA analysis software system. version 7. São Caetano do Sul: Statsoft Inc., 2004.

GOLDEN, B.L.; SNELLING, W.M.; MALLINCKRODT, C.H. User's guides and reference manual. Fort Collins: Colorado State University, 1995. (Tech. Bulletin LTB92-2)

HAIR, J.R.; ANDERSON, R.E.; TATHAM, R.L. et al. Análise multivariada de dados. Porto Alegre: Buckman, 2005. 593p.

HARTIGAN, J.A. Clustering algorithms. New York: Wiley, 1975. 351p.

JOHNSON, R.A.; WICHERN, D.W. Applied multivariate statistical analysis. 2.ed. Englewood Cliffs: Prentice-Hall, 1992. 642p.

KIRKPATRIC, M.; MEYER; K. Direct estimation of genetic principal components: simplified analysis of complex phenotypes. Genetics, v.168, p.2295-2306, 2004.

LOBO, R.B.; BEZERRA, L.A.F.; OLIVEIRA, H.N. et al. Avaliação genética de animais jovens, touros e matrizes. Ribeirão Preto: GEMAC/FMRP/USP, 2003. 86p.

MASCIOLI, A.S.; FARO, L.; ALENCAR, M.M. et al. Estimativas de parâmetros genéticos e fenotípicos e análise de componentes principais para características de crescimento na raça Canchim. Rev. Bras. Zootec., v.29, p.1654-1660, 2000.

PÉREZ, C. Técnicas estadísticas con SPSS. Madrid: Pearson Educación, 2001, 438p.

REIS, E. Estatística multivariada aplicada. Lisboa: Edições Sílabo, 2001. 343p.

TONHATI, H.; MARCONDES, C.R.; LÔBO, R.B. Sumários e Aplicações. In: WORKSHOP SELEÇÃO EM BOVINOS DE CORTE, 5., 2003, Salvador. Anais..., Salvador: [s.n.], 2003.

VOZZI, P.A. Análise da estrutura e variabilidade genética dos rebanhos do Programa de Melhoramento Genético da Raça Nelore. 2004. 62f. Dissertação (Mestrado) - Faculdade de Medicina de Ribeirão Preto, Universidade de São Paulo, Ribeirão Preto. 